\title{
A Perturbation and Observation Approach to Decentralized Power Loss Minimization in AC Microgrids
}

\author{
Nestor Vazquez, Samson Shenglong Yu, Tat Kei Chau, Tyrone Fernando, Herbert Ho-Ching Iu \\ Department of Electrical, Electronic and Computer Engineering \\ The University of Western Australia, Perth, Australia \\ nestor.vazquezbarajas@research.uwa.edu.au and samson.yu@uwa.edu.au
}

\begin{abstract}
This study presents a simple decentralized strategy for power loss minimization in transmission lines in isolated AC microgrids. In this scenario, the proposed approach works as a decentralized secondary control, since the generation units use droop functions as primary controllers (looking at the microgrid from a hierarchical point of view). Therefore, assuming the droop control guarantees the power balance between generation and consumption (as long as the total power demand does not exceed the microgrid generation capacity), the proposed secondary control has the objective of reducing the power loss in the transmission lines. This secondary control adapts the primary control droop functions in order to reach a steady state where the total power loss is minimum. The main advantage of this strategy is the completely autonomous operation of the participating DGs, using only local measurements of system frequency and bus voltages. The distributed adaptation of the droop functions for each of the DGs is carried out by performing a Perturbation and Observation (P\&O) algorithm. Initially, for each DG at a time, a small perturbation in the $P-f$ droop function is applied. Then, the produced effects on the system frequency and bus voltage are examined in order to identify if the resultant power balance has reduced or increased the system power loss. This procedure is executed indefinitely by all the participating DGs in the microgrid over a fixed time-step. Eventually, the system reaches a steady state where the power generation of each DG produce the minimum power loss in the transmission lines. The procedure continues endlessly, and any load variation or change in the microgrid topology will lead to a new steady state with different power generation levels for each DG and minimum power loss.
\end{abstract}

Index Terms-Microgrid, decentralized control, power loss minimization, droop control.

\section{INTRODUCTION}

The studies on Microgrids have become a trend in recent years, trying to provide reliable and robust techniques for including the many different types of Distributed Energy Resources (DERs) [1]. Designing controllers for microgrids presents different problems than those found on the traditional power grid, regarding to system stability, optimality and power balance. Particularly in the case of system optimality, different problems can be presented, e.g., minimization of the power generation cost, minimization of power loss in the transmission

This work is supported by the Australian Research Council (ARC) Discovery Project (DP) grant, DP170104426. lines, minimization of greenhouse gas emission, maximization of the battery life expectancy, etc. In the particular case of the isolated Microgrid, the slack bus participating as a power generator with virtually infinite capacity is absent, and the power balance must be maintained by all the distributed generation units. This inevitably represents a limitation on the loading capabilities of the system. In these scenarios, the power demand must be proportionally shared between the DGs, using droop functions as load sharing mechanisms. This strategy is commonly used, taking advantage of the completely decentralized operating mode in which only local measurements of system frequency and bus voltages are needed [2]-[4]. Different strategies for improving the power sharing in droop controlled isolated microgrids have been widely proposed [5]-[9]. Regarding to the optimization problem in isolated microgrids, researchers have proposed the construction of optimal droop functions in order to fulfill specific objectives. For example, the authors in [10] proposed the construction of the droop functions based on the fuel consumption of the DGs. In order to minimize the generation cost of the system, the authors in [11] and [12] proposed an alternative to construct the droop functions. Non-linear and dynamic droop functions have also been presented as a way to improve the optimization outcomes in [13]-[16]. On the other hand, the power loss minimization problem in the isolated microgrid gains importance since the power balance must be maintained solely by the DGs within the system, and there is not a slack bus capable of supplying any load increase. This problem is addressed in [15], where non-linear droop functions are built to minimize the power loss, however this approach has the drawback of needing an off-line initial tuning procedure. Another approach is presented in [17], where the power loss minimization problem is addressed by designing a decentralized voltage control strategy for the DGs which adjust the output voltage of the inverters, but droop-based DGs are not considered.

A completely decentralized optimization approach for minimizing power loss in isolated microgrids is presented in this paper. Typically, the DGs within an isolated microgrid have a droop function in its primary level control. This control is widely implemented because of its simplicity and 


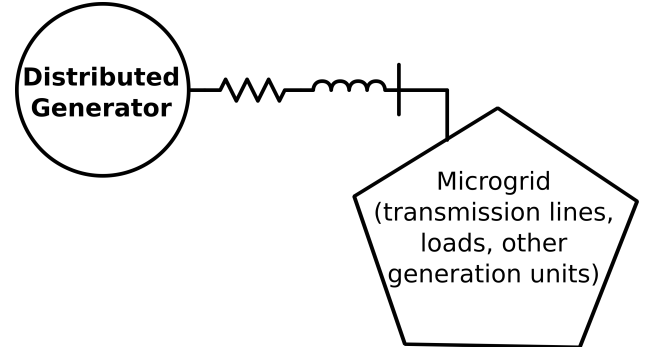

Fig. 1: Distributed Generator connected to a microgrid.

robustness. The depiction of the isolated microgrid studied in this paper is shown in Fig. 1, where the DGs are unaware of the topology and size of the system. As stated before, the DGs in this study are presented with a droop control in its primary level, in charge of sharing proportionally the system load and maintaining the power balance [3], [5], [6]. The proposed strategy, with a larger time scale and the objective of minimizing the power loss, accounts for the secondary level control. The main advantage of the proposed strategy is the completely decentralized manner in which the secondary control is implemented, using solely local measurements of bus voltage and system frequency. This removes the need of using an advanced communication system for controlling the generation units. The adaptability of the droop functions is performed by using a Perturbation and Observation (P\&O) approach, usually implemented in Maximum Power Point Tracking (MPPT) algorithms. The presented strategy relies on the manipulation of the droop functions of the DGs, in order to search for a steady state where the power loss is minimized. If any change in the loads or topology of the grid is presented, the system will search for the new steady state with minimum power loss. This strategy is easy to implement and does not need data communication between the generation units, showing its high suitability for real world applications.

The remainder of the paper is organized as follows. In Section II the decentralized primary droop controller for the DGs is shortly described, showing the droop functions of the power controller. In Section III the proposed secondary controller is presented, describing the power loss minimization strategy. The case studies with simulation results are presented in section IV, showing the performance of the power loss minimization strategy. A conclusion is presented in Section V.

\section{Primary Control}

The linear droop control is the most common primary level control strategy of DGs used in isolated Microgrids, which has been widely adopted due to its simplicity and robustness. It utilizes the frequency and voltage deviations caused by load variations to construct the control signals, in order to compute the power dispatch. The classical relations between real power and frequency, and reactive power and voltage can be expressed as [3]:

$$
f=f_{\max }-m_{D G} \cdot P_{D G},
$$

$$
V=V_{\max }-n_{D G} \cdot Q_{D G},
$$

where $f_{\max }$ and $V_{\max }$ are the maximum frequency and bus voltage of the Microgrid and $P_{D G}$ and $Q_{D G}$ are the active and reactive powers injected by the DG. $m_{D G}$ and $n_{D G}$ are the droop coefficients, computed from:

$$
\begin{aligned}
& m_{D G}=\frac{f_{\max }-f_{\min }}{P_{D G}^{c a p}}, \\
& n_{D G}=\frac{V_{\max }-V_{\min }}{Q_{D G}^{c a p}},
\end{aligned}
$$

where $f_{\min }$ and $V_{\min }$ are the minimum frequency and voltage of the Microgrid and $P_{D G}^{c a p}$ and $Q_{D G}^{c a p}$ are the active and reactive power capacities of the DG. Fig. 2 shows the $P-f$ and $Q-V$ linear relations for a DG in a Microgrid.

The main advantage of this control is that it does not require a communication system to achieve the active power load sharing, since the Microgrid frequency can provide sufficient information for computing the active power dispatch of each DG as a function of its capacity.
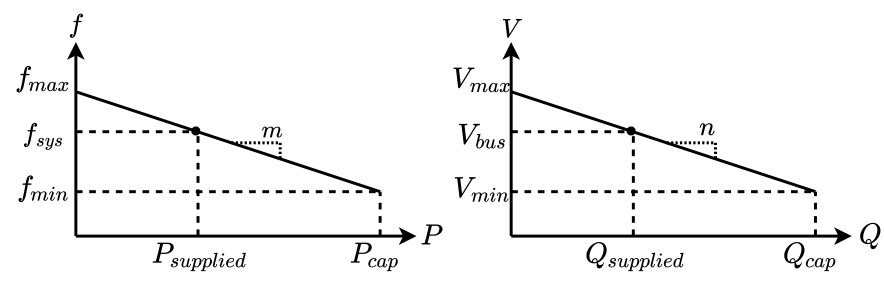

Fig. 2: Droop functions for Active and Reactive powers

\section{Proposed Decentralized Secondary Control}

The power balance is guaranteed by the primary level control embedded in each DG, using droop functions to compute the active and reactive power dispatch. Thus, the presented optimization strategy can then be classified as secondary control (from a hierarchical point of view), since it operates in a larger time scale and produces the droop setpoints for the primary level controllers. Usually, secondary level controllers in isolated microgrids are designed with the objective of restoring bus voltages and system frequency (finding the setpoints where $f_{\text {sys }}=f_{\text {nom }}$ and $V_{\text {bus }}=V_{\text {nom }}$ ) [7]. However, the objective of the strategy presented in this paper is to minimize the power loss in the transmission lines, without compromising the system stability (maintaining deviations of system frequency less than $\pm 1 \mathrm{~Hz}$ and voltage less than $\pm 5 \%$ ).

As long as the power generation capacities of the microgrid are not exceeded, the power balance can be satisfied and the power demand is shared among all the DGs within the isolated system, i.e.,

$$
\sum_{k=1}^{L} P_{\text {Gen }_{k}}=\sum_{j=1}^{M} P_{\text {Load }_{j}}+\sum_{r=1}^{N} P_{\text {Loss }_{r}},
$$

where $P_{G e n_{k}}$ is the active power supplied by the generation unit $k, P_{\text {Load }_{j}}$ is the active power dragged by the load $j$ and 
$P_{\text {Loss }_{r}}$ is the active power dissipated by the transmission line $r ; L$ is the number of generation units, $M$ is the number of load buses and $N$ is the number of transmission lines.

\section{A. Load and Power Loss}

The presented secondary controller can be conceptualized from the construction of a Load + Loss profile, depicted in Fig. 3 as the green line. The Load + Loss profile is theorized by gradually incorporating a constant offset $\Delta f_{\text {step }}$ in the droop function of a DG; as a result, the generated power of such unit changes and supplies a corresponding part of the system total Load + Loss (because the total power demand is shared among all the generation units with droop control). Assuming that the total load of the system is constant or changes slowly over time, the red line in Fig. 3 represents the corresponding part of the total load that such DG supplies. Consequently, the curvature in the green line represents the differences in power loss, and the closest point of the Load + Loss profile to the load profile indicates the point with minimum system power loss for that DG. Fig. 3 was theoretically plotted to illustrate the procedure of the gradual incorporation of the constant offset in the droop function of a DG, and its change in output power; $f_{\max }$ and $f_{\min }$ stand for the maximum deviation of the system frequency, $\pm 1 H z ; f_{\text {sys }}$ is the steady state system frequency. Despite the changes in the offsets and deviation limits, the system frequency is always maintained within $\pm 1 \mathrm{~Hz}$ range of the nominal value.

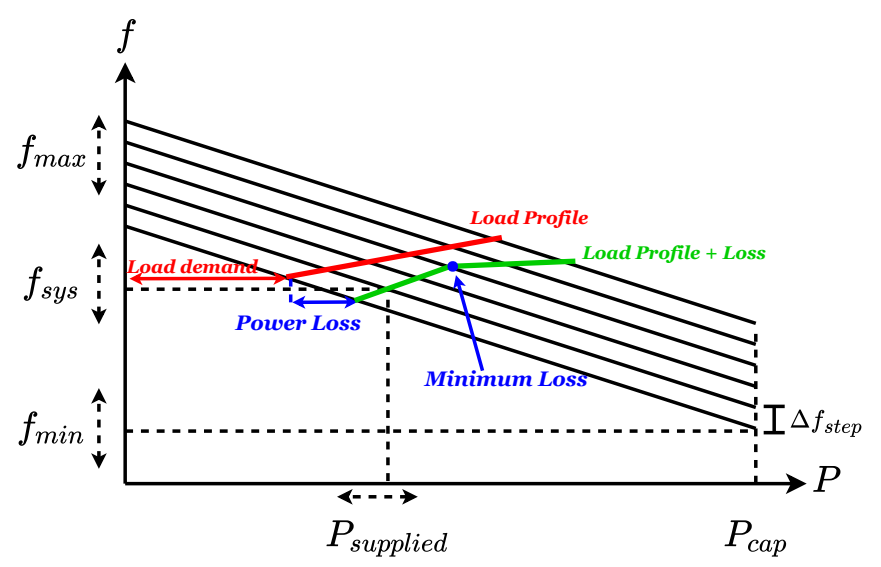

Fig. 3: Load profile for a Distributed Generator

\section{B. Perturbation Step}

For each DG, the perturbation step is performed by adding a fixed offset $\Delta f_{\text {step }}$ in its droop function, which produces a change in its generated power. Therefore, the system reaches a new steady state frequency, and new power levels for all the DGs with droop control in the microgrid. The new power level supplied by the DG $k$ once the perturbation has been applied in its droop function is expressed as follows,

$$
P_{k}^{\prime}=\frac{f_{k}^{\max }+\Delta f_{\text {step }}-f_{\text {sys }}}{m_{k}} .
$$

\section{Observation and Decision Making Step}

After the perturbation step is performed, the DG must identify the direction ( $\Delta f_{\text {step }}$ whether positive or negative) in which the search for the optimal offset must be carried on. Due to the lack of information each DG has about the topology and load demand, it is not possible to identify if the power loss was increased or decreased; but, analyzing the normalized system frequency and active power rates of change, it is plausible to choose the sign of the offset for the next perturbation step.

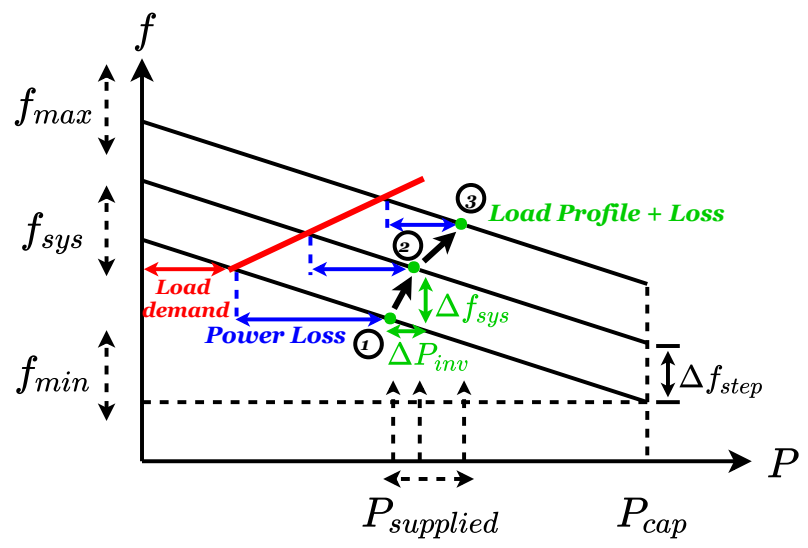

Fig. 4: Conceptual illustration of the adaptation procedure of a generation unit.

The process of adding the perturbations in the droop function of a DG is shown in Fig. 4, where numbers 1, 2 and 3 correspond to the initial point and two perturbation steps, respectively. The chronological implementation of the secondary controller in a DG is shown by the arrows, from 1 to 3 .

In Fig. 3 is shown that, assuming that the loads in the system are constant or change slowly over time, the curve of the Load + Loss profile (represented by the green line) corresponds to a difference in the system power loss. Therefore, the point where the power loss is minimum corresponds to the point where the Load + Loss profile is closer to the Load profile. The exploration of this function is blind and not possible to estimate, thus the secondary control embedded in each DG must perform a blind search for this point over tu curve. In order to reach the optimal offset, each DG uses the rate of change of the system frequency and active power (functions where the optimal point can also be observed), as shown in Fig. 5. Equations (7) and (8) are computed to locate the optimal offset, where $f_{s y s}$ and $P_{k}$, and $f_{s y s}^{\prime}$ and $P_{k}^{\prime}$ are the system frequencies and active powers generated before and after the perturbation is performed by the generation unit $k$. Term $\Delta t$ is the time step of secondary controller.

$$
\begin{aligned}
\frac{\partial f_{s y s}}{\partial t} & =\frac{f_{s y s}^{\prime}-f_{s y s}}{\Delta t} \\
\frac{\partial P_{k}}{\partial t} & =\frac{P_{k}^{\prime}-P_{k}}{\Delta t}
\end{aligned}
$$



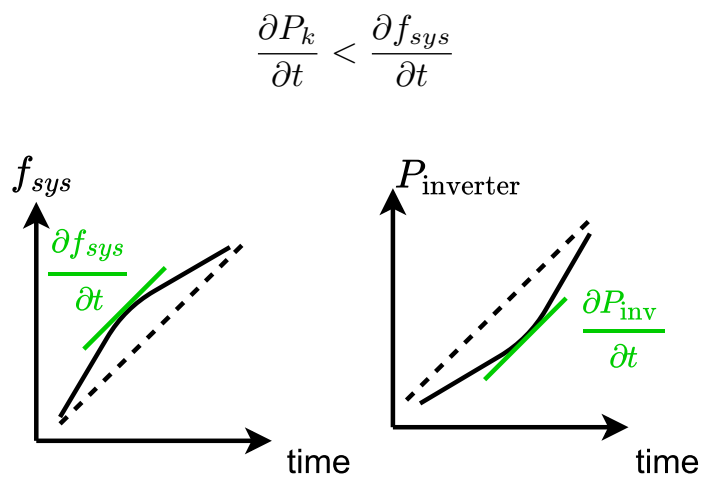

Fig. 5: Derivatives of frequency and active power.

In order to rule the execution times of the distributed optimization procedures, a real time counter is embedded in the controllers of each DG. Fig. 6 depicts graphically and chronologically the sequences, where $T_{\text {count }}$ is the real time counter and $T_{\text {adapt }}$ is the time signal that drives the implementation of the optimization procedure. The control sequences (where $\Delta t$ is the time step that rules the $\mathrm{P} \& \mathrm{O}$ procedures and $\Delta T$ is the total adaptation procedure time step) are shown in Fig. 7.

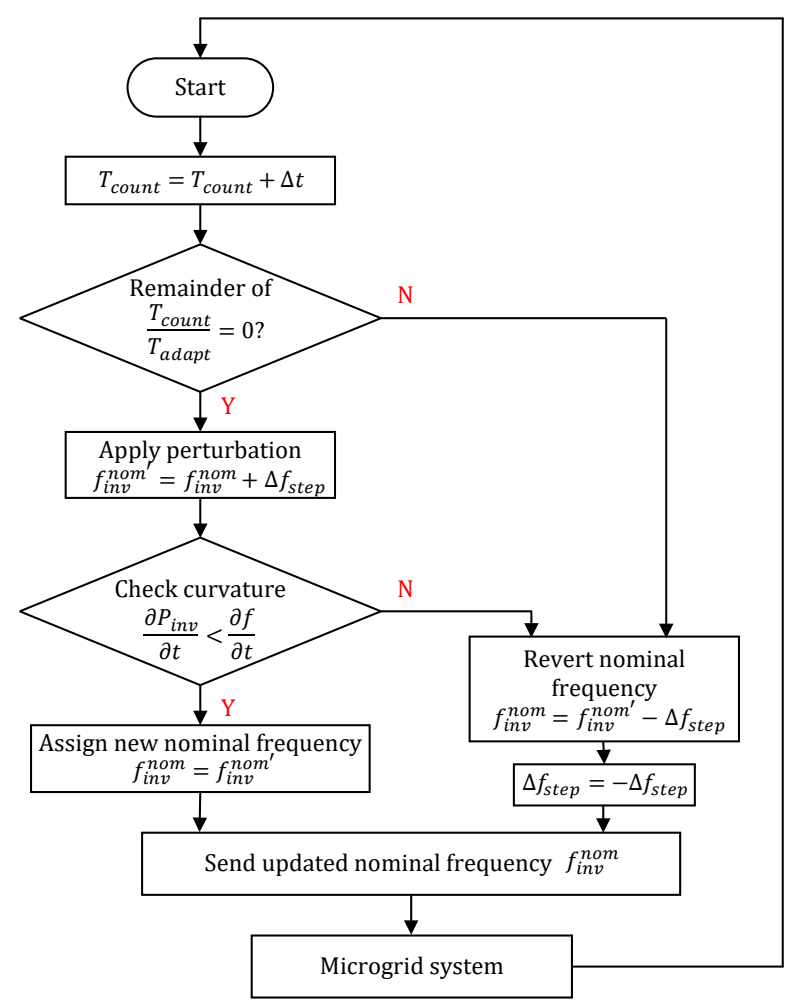

Fig. 6: Embedded secondary control.

\section{CAse Study}

In order to validate the performance of the presented power loss minimization secondary controller, an isolated microgrid model coded in MATLAB was developed, with droop

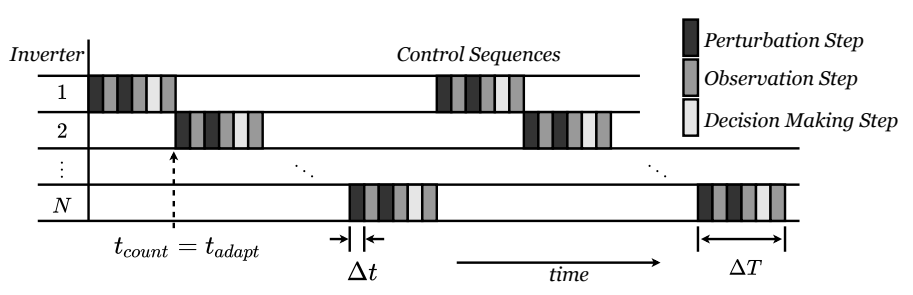

Fig. 7: Distributed generators control sequences.

functions as primary controllers of the DGs. The proposed optimization strategy is embedded in each generation unit. The topology of the test system is depicted in Fig. 8, and the power lines characteristics are shown in TABLE I. The time steps of the optimization procedures are fixed.

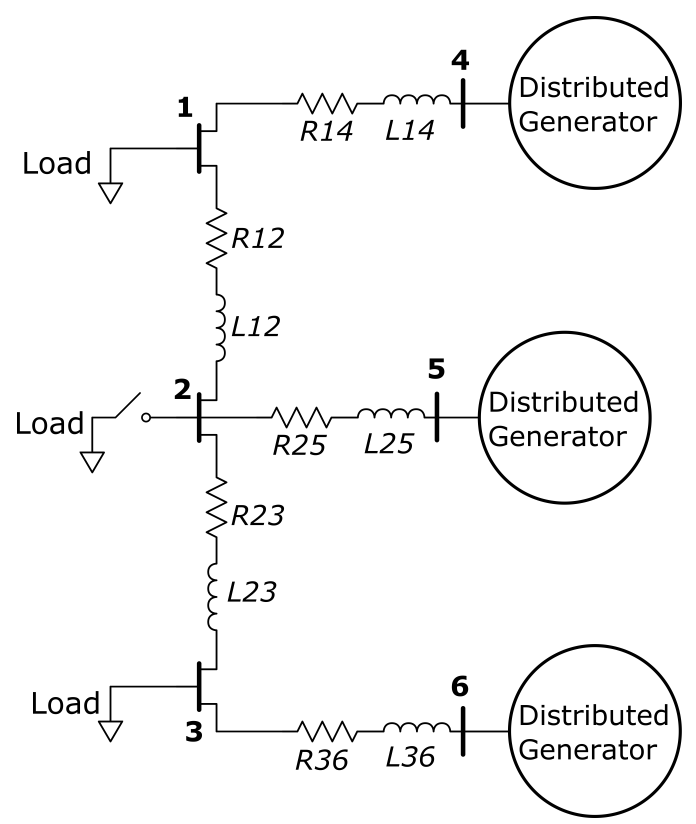

Fig. 8: Topology of the studied Microgrid.

TABLE I: Line parameters

\begin{tabular}{llll}
\hline From & To & $R(\Omega)$ & $L(\mathrm{mH})$ \\
\hline 1 & 2 & 0.63 & 0.318 \\
\hline 1 & 4 & 0.5 & 0.35 \\
\hline 2 & 3 & 0.35 & 1.843 \\
\hline 2 & 5 & 0.4 & 0.25 \\
\hline 3 & 6 & 0.25 & 0.05 \\
\hline
\end{tabular}

The performance of the presented strategy is tested in two case studies, where its feasibility is verified. The first case scenario shows a load variation, which proves that the secondary controller is able to cope with changes in the power demand. The second case shows a change in the topology of the simulated microgrid. The operation of the optimization strategy is confirmed by the simulation results. TABLE II shows the time and frequency steps ruling the execution of the secondary controller. Since the objective of this study is to investigate the feasibility of the presented strategy, these 
values are fixed and selected appropriately to guarantee the microgrid stability.

TABLE II: Control parameters

\begin{tabular}{ll}
\hline$\Delta t$ & $0.5 \mathrm{~s}$ \\
\hline$\Delta f_{\text {step }}$ & $0.01 \mathrm{~Hz}$ \\
\hline
\end{tabular}

\section{A. Case 1}

For this case, loads and DGs characteristics are displayed in TABLE III, where the arrow symbols $\nearrow$ and $\searrow$ indicate that the load is increasing or decreasing, respectively. Loads slowly change from $t=400 \mathrm{~s}$ to $t=500 \mathrm{~s}$, in order to test the performance of the strategy during changing circumstances: load at bus 1 is increased to $6.3 \mathrm{~kW}$ and $2.5 \mathrm{kVAR}$, while load at bus 3 is reduced to $3.9 \mathrm{~kW}$ and $1.2 \mathrm{kVAR}$. The simulation plots for this case are shown in Figs. 9 (a) (d), accounting for active power generation, power loss, frequency and busbar voltages, respectively. Fig. 9 (b) shows the power loss reduction in the grid when using the proposed secondary control, compared to the power loss of the grid when classic droop control is used.

TABLE III: Case 1 loads and DGs (kW, kVAR)

\begin{tabular}{llll}
\hline Bus & $P_{\text {Load }}$ & $Q_{\text {Load }}$ & $\begin{array}{l}\text { DG } \\
\text { Capacity }\end{array}$ \\
\hline 1 & $3.435 \nearrow$ & $1.548 \nearrow$ & - \\
\hline 2 & 0 & 0 & - \\
\hline 3 & $6.842 \searrow$ & $2.204 \searrow$ & - \\
\hline 4 & - & - & 10 \\
\hline 5 & - & - & 10 \\
\hline 6 & - & - & 10 \\
\hline
\end{tabular}

\section{B. Case 2}

For this case the total load of the grid is slightly increased and load in bus number 2 is connected (which leads to a completely different initial steady state for bus voltages, active powers and system frequency). Loads and DGs characteristics are mentioned in TABLE IV, where the arrow symbols $\nearrow$ and $\searrow$ indicate that the load is increasing or decreasing, respectively. Again, loads slowly change in time from $t=400 \mathrm{~s}$ to $t=500 \mathrm{~s}$, testing the performance of the strategy under changing circumstances: load in bus number 1 reaches 5.1 $\mathrm{kW}$ and $1.7 \mathrm{kVAR}$, load in bus number 2 reaches 3.485 $\mathrm{kW}$ and $1.195 \mathrm{kVAR}$ and load in bus number 3 is reduced to $4.3 \mathrm{~kW}$ and $1.5 \mathrm{kVAR}$. The simulation plots for this case are depicted in Figs. 10 (a) (d), accounting for active power generation, power loss, frequency and busbar voltages, respectively. Fig. 10 (b) shows the power loss reduction in the grid when using the proposed secondary control, compared to the power loss of the grid when classic droop control is used.

\section{CONClusion}

This study proposes an strategy for reducing the power loss in isolated microgrids. The main advantage of this approach is the completely decentralized operation of the participating units, which do not need information about the microgrid

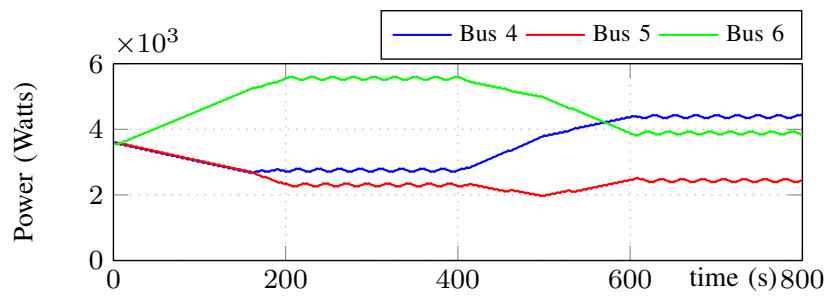

(a) Active power from generation units

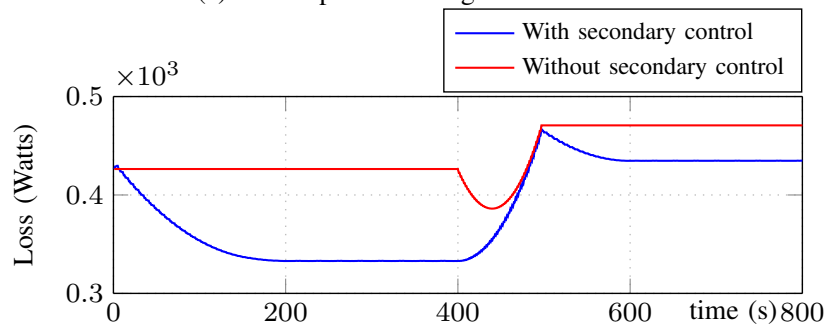

(b) Power loss of microgrid

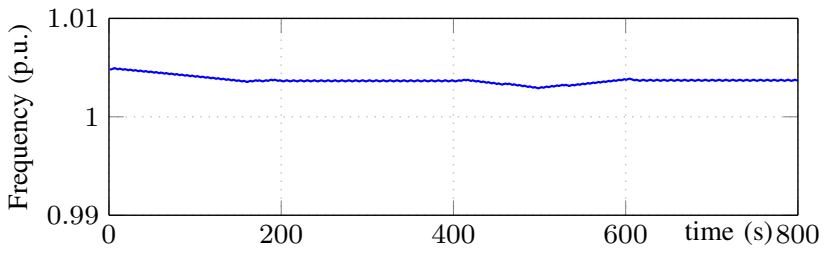

(c) System frequency

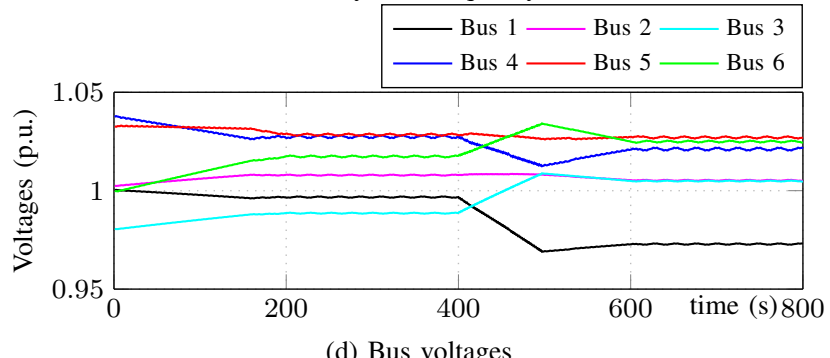

Fig. 9: Simualtion resuslts in case 1

TABLE IV: Case 2 loads and DGs (kW, kVAR)

\begin{tabular}{llll}
\hline Bus & $P_{\text {Load }}$ & $Q_{\text {Load }}$ & $\begin{array}{l}\text { Inverter } \\
\text { Capacity }\end{array}$ \\
\hline 1 & $4.435 \nearrow$ & $1.548 \nearrow$ & - \\
\hline 2 & $2.015 \nearrow$ & $0.705 \nearrow$ & - \\
\hline 3 & $5.851 \searrow$ & $2.003 \searrow$ & - \\
\hline 4 & - & - & 10 \\
\hline 5 & - & - & 10 \\
\hline 6 & - & - & 10 \\
\hline
\end{tabular}

topology or the size of loads. This approach, embedded within the participating units, uses local measurements of frequency and voltage to identify whether to increase or decrease the active power injected into the grid. Simulations have shown that the approach is able to reduce power loss and keep bus voltages and system frequency within functional levels, in different loading scenarios. Nonetheless, the performance of the presented strategy declines when the topology of the microgrid becomes more complex, since it depends on a blind exploration of unknown functions: the Load + Loss profiles. 


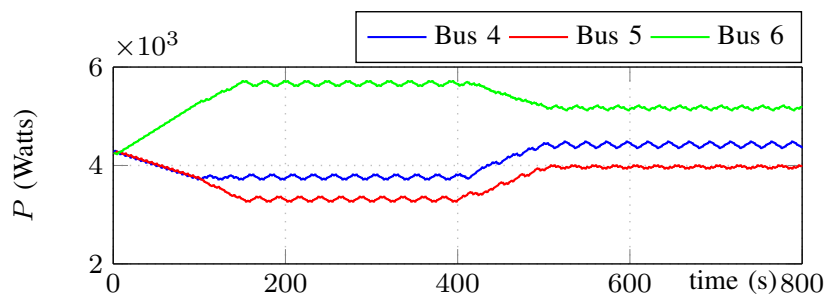

(a) Active power from generation units

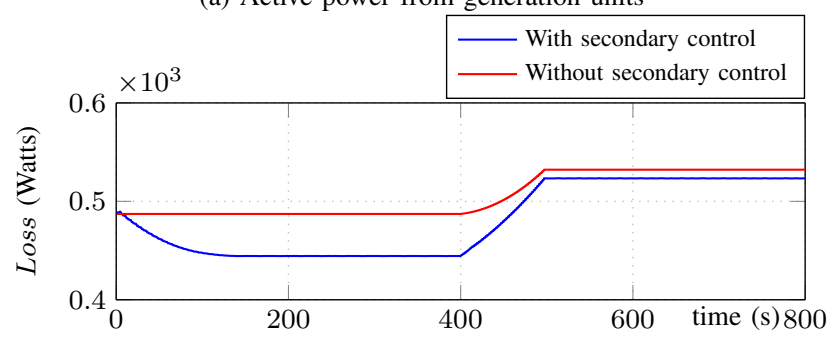

(b) Power loss of microgrid

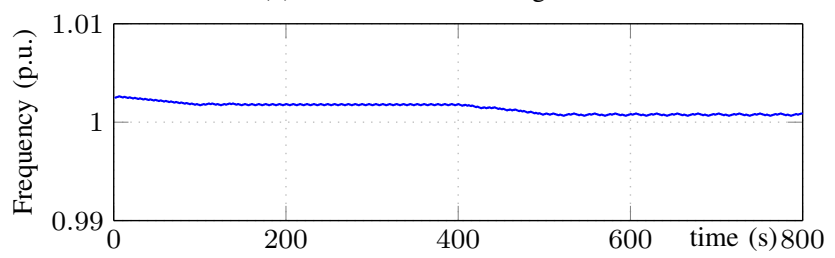

(c) System frequency

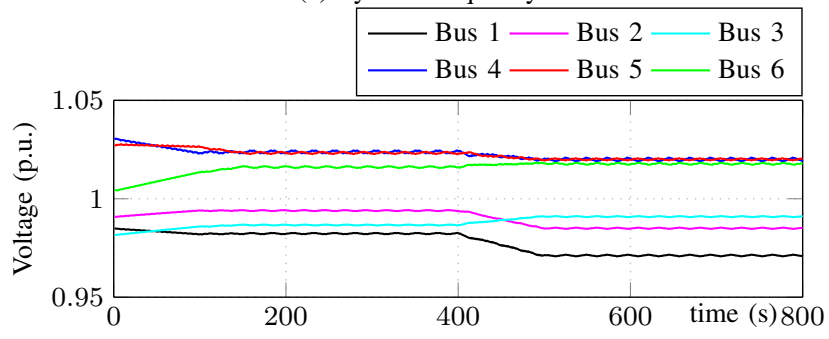

(d) Bus voltages

Fig. 10: Simualtion resuslts in case 2

Regarding to this topic, future studies may cover system stability analysis, fully decentralized topology identification (in order to improve the performance when the system becomes more complex) and the inclusion of other types of generation units.

\section{REFERENCES}

[1] N. Hatziargyriou, H. Asano, R. Iravani, and C. Marnay, "Microgrids," IEEE Power and Energy Magazine, vol. 5, no. 4, pp. 78-94, July 2007.

[2] D. E. Olivares, A. Mehrizi-Sani, A. H. Etemadi, C. A. Caizares, R. Iravani, M. Kazerani, A. H. Hajimiragha, O. Gomis-Bellmunt, M. Saeedifard, R. Palma-Behnke, G. A. Jimnez-Estvez, and N. D Hatziargyriou, "Trends in microgrid control," IEEE Transactions on Smart Grid, vol. 5, no. 4, pp. 1905-1919, July 2014.

[3] Y. Han, H. Li, P. Shen, E. A. A. Coelho, and J. M. Guerrero, "Review of active and reactive power sharing strategies in hierarchical controlled microgrids," IEEE Transactions on Power Electronics, vol. 32, no. 3, pp. 2427-2451, March 2017.

[4] J. M. Guerrero, J. C. Vsquez, and R. Teodorescu, "Hierarchical control of droop-controlled dc and ac microgrids: a general approach towards standardization," in 2009 35th Annual Conference of IEEE Industrial Electronics, Nov 2009, pp. 4305-4310.
[5] K. D. Brabandere, B. Bolsens, J. V. den Keybus, A. Woyte, J. Driesen, and R. Belmans, "A voltage and frequency droop control method for parallel inverters," IEEE Transactions on Power Electronics, vol. 22, no. 4, pp. 1107-1115, July 2007.

[6] Z. Ahmad and S. N. Singh, "Droop control strategies of conventional power system versus microgrid based power systems - a review," in 2015 International Conference on Computational Intelligence and Communication Networks (CICN), Dec 2015, pp. 1499-1504.

[7] H. Bevrani and S. Shokoohi, "An intelligent droop control for simultaneous voltage and frequency regulation in islanded microgrids," IEEE Transactions on Smart Grid, vol. 4, no. 3, pp. 1505-1513, Sept 2013.

[8] P. C. Loh, D. Li, Y. K. Chai, and F. Blaabjerg, "Autonomous operation of hybrid microgrid with ac and dc subgrids," IEEE Transactions on Power Electronics, vol. 28, no. 5, pp. 2214-2223, May 2013.

[9] A. Solanki, A. Nasiri, V. Bhavaraju, Y. L. Familiant, and Q. Fu, "A new framework for microgrid management: Virtual droop control," IEEE Transactions on Smart Grid, vol. 7, no. 2, pp. 554-566, March 2016.

[10] C. A. Hernandez-Aramburo, T. C. Green, and N. Mugniot, "Fuel consumption minimization of a microgrid," IEEE Transactions on Industry Applications, vol. 41, no. 3, pp. 673-681, May 2005.

[11] F. Chen, M. Chen, Q. Li, K. Meng, Y. Zheng, J. M. Guerrero, and D. Abbott, "Cost-based droop schemes for economic dispatch in islanded microgrids," IEEE Transactions on Smart Grid, vol. 8, no. 1, pp. 63-74, Jan 2017.

[12] I. U. Nutkani, P. C. Loh, P. Wang, and F. Blaabjerg, "Linear decentralized power sharing schemes for economic operation of ac microgrids," IEEE Transactions on Industrial Electronics, vol. 63, no. 1, pp. 225-234, Jan 2016.

[13] F. Cingoz, A. Elrayyah, and Y. Sozer, "Plug-and-play nonlinear droop construction scheme to optimize islanded microgrid operations," IEEE Transactions on Power Electronics, vol. 32, no. 4, pp. 2743-2756, April 2017.

[14] A. Elrayyah and Y. Sozer, "Construction of nonlinear droop relations to optimize islanded microgrids operation," in 2013 IEEE Energy Conversion Congress and Exposition, Sept 2013, pp. 1663-1668.

[15] Q. T. T. Tran, H. Shehadeh, E. R. Sanseverino, S. Favuzza, and M. L. D. Silvestre, "Nonlinear droop control for minimum power losses operation in islanded microgrids," in 2017 IEEE International Conference on Environment and Electrical Engineering and 2017 IEEE Industrial and Commercial Power Systems Europe (EEEIC / I CPS Europe), June 2017, pp. 1-5.

[16] H. Mahmood, D. Michaelson, and J. Jiang, "Reactive power sharing in islanded microgrids using adaptive voltage droop control," IEEE Transactions on Smart Grid, vol. 6, no. 6, pp. 3052-3060, Nov 2015.

[17] C. Ahn and H. Peng, "Decentralized voltage control to minimize distribution power loss of microgrids," IEEE Transactions on Smart Grid, vol. 4, no. 3, pp. 1297-1304, Sept 2013. 